\title{
Osteocondritis de sesamoideos: Revisión
}

\author{
Hallucal sesamoid osteochondritis: Review \\ Javier Navarro Marruedo ${ }^{1}$, José Luis Muñoz Sánchez ${ }^{1}$ \\ ${ }^{1}$ Graduado en Podología. \\ javinavarro46@gmail.com \\ joseguis16@hotmail.com
}

Correspondencia:

Javier Navarro Marruedo

Vá Ibérica 2, Blq 5, Esc 4, $2^{\circ} \mathrm{A}$.

50009, Zaragoza

Correo-e: javinavarro46@gmail.com

Fecha de recepción: 9 julio de 2014

Fecha de aceptación: 28 de octubre de 2014

Los autores declaran no tener ningún tipo de interés económico o comercial.

\section{RESUMEN}

La osteocondritis de los sesamoideos es una enfermedad infrecuente, que se puede dar en cualquiera de los dos sesamoideos, siendo una patología incapacitante. A pesar de que los sesamoideos juegan un papel fundamental en la mecánica del antepié, algunos trastornos que se dan en ellos a menudo se pasan por alto o son mal diagnosticados.

Se revisa y analizan las características clínicas de la enfermedad, su tratamiento y las claves diagnósticas que nos permiten establecer un correcto diagnóstico diferencial con otros procesos patológicos que afectan a los sesamoideos.

Palabras clave: Osteocondritis; sesamoideos; necrosis avascular; tratamiento.

\begin{abstract}
Osteochondritis of the sesamoid is a rare disease, which can result in either sesamoid being disabling pathology. Despite the fact the hallucal sesamoids play a crucial role in forefoot mechanics, pathology resulting from them are often overlooked or misdiagnosed.

We review and analyze the clinical characteristics of the disease, its treatment and diagnostic clues that allow us to establish a correct differential diagnosis with other pathological processes affecting the sesamoid.
\end{abstract}

Key words: Osteochondritis; sesamoids; avascular necrosis; treatment.

Referencia normalizada: Navarro Marruedo, J., Muñoz Sánchez, J.L. Osteocondritis de sesamoideos: Revisión. Rev. Int. Cienc. Podol. 2016; 10(1): 17-25.

Sumario: 1. Introducción. 2. Anatomía y desarrollo embiológico. 3. Vascularización. 4. Biomecánica. 5. Etiología. 6. Diagnóstico y diagnóstico diferencial. 7. Tratamiento. 8. Conclusión. Bibliografía. 


\section{INTRODUCCIÓN}

Se presenta una revisión bibliográfica sobre la osteocondritis de sesamoideos describiendo su etiología, fisiopatología, diagnóstico y tratamiento. El objetivo de esta revisión es exponer de forma sintetizada la evidencia científica en relación a esta patología. Para ello se buscan en las bases de datos Pubmed, Cochrane Libray, BUCea, Enfispo y Catálogo Cisne desde Diciembre de 2013 hasta Abril de 2014, utilizando las palabras clave de la Tabla 1 .

Se incluyen revisiones, revisiones sistemáticas, y casos clínicos de los últimos diez años. Dada la poca cantidad de literatura encontrada, es necesario realizar otra búsqueda sin limitar los términos de la misma utilizando términos más específicos, y ampliando la fecha de publicación. Se revisaron en total quinientos veinticinco artículos, de los cuales se excluyen cuatrocientos cuarenta y ocho según los criterios de inclusión/exclusión de la tabla 2. Posteriormente se realiza una lectura del texto completo y se consideran relevantes veinte artículos que se incluyen en la revisión, como se muestra en el Grafico 1.

El término osteocondritis se ha utilizado para describir un grupo de patologías que afectan al esqueleto en crecimiento. Estas patologías se dan como resultado de un crecimiento anormal, daño, o sobreuso de los núcleos primarios y secundarios de crecimiento. La etio-

\begin{tabular}{|c|c|c|c|c|}
\hline Términos de búsqueda & Pubmed & Medline & Cochrane & BUCM \\
\hline $\begin{array}{l}\text { Osteochondritis and } \\
\text { sesamoid }\end{array}$ & 17 & 0 & 0 & 66 \\
\hline Osteochondritis and foot & 53 & 3 & 1 & $\Xi$ \\
\hline $\begin{array}{l}\text { Avascular necrosis and } \\
\text { sesamoid }\end{array}$ & 12 & 0 & 0 & 80 \\
\hline $\begin{array}{l}\text { Osteonecrosis and se- } \\
\text { samoid }\end{array}$ & 16 & 0 & 0 & 118 \\
\hline $\begin{array}{l}\text { Osteochondritis and } \\
\text { sesamoid and treatment }\end{array}$ & 3 & 0 & 0 & 94 \\
\hline
\end{tabular}

Tabla 1. Resultados de la estrategia de búsqueda.

\begin{tabular}{|ll|}
\hline Criterios de inclusión & Criterios de exclusión \\
\hline - Revisión & - Artículos que no incluyan tratamiento \\
$\bullet$ Revisión sistemática & - Artículos no publicados en inglés \\
$\bullet$ Casos clínicos & - Opinión del autor \\
& - Reseñas de libros \\
$-<$ de 10 años & - No contar con el artículo en texto completo, aunque \\
& cumpliera criterios de inclusión \\
& - Artículos de veterinaria
\end{tabular}

Tabla 2. Criterios de inclusión/exclusión. 
Gráfico 1. Revisión de la literatura.

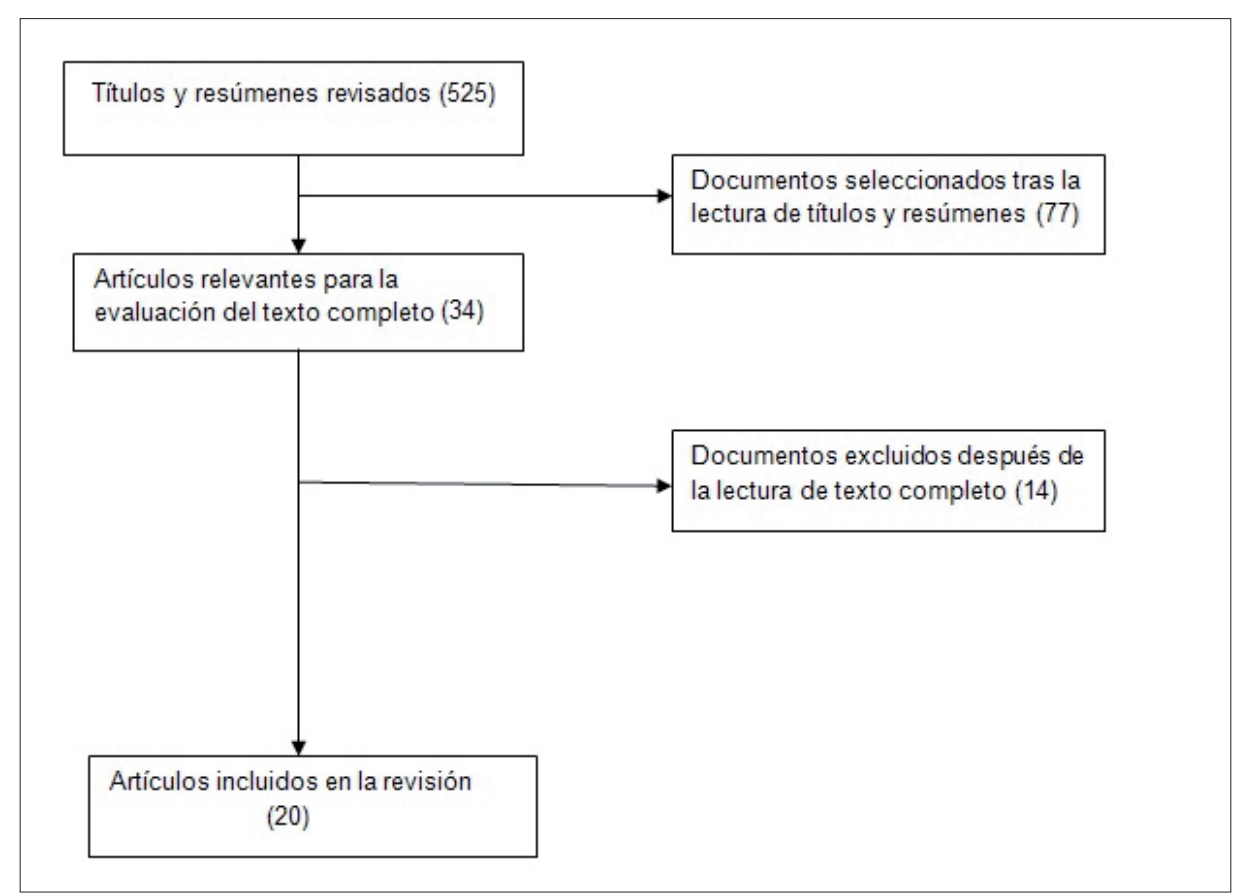

logía exacta de las osteocondritis se desconoce, pero se piensa que puede estar relacionado con la genética, los traumatismos repetitivos, patología vascular asociada, factores mecánicos y los desajustes hormonales ${ }^{1}$.

La osteocondritis de los sesamoideos o necrosis avascular fue descrita por primera vez por Renander con dos casos en el sesamoideo medial en $1924^{2}$ y continúa considerándose como infrecuente e incompletamente definida. Desde entonces la literatura relacionada con esta patología ha consistido en series de casos clínicos.

Afecta con mayor frecuencia a personas que practican ballet o deportes de salto (balonmano, baloncesto, aeróbic, atletismo) habiéndose encontrado en estas poblaciones una incidencia de la enfermedad del 0,2\%. La edad más habitual de presentación del cuadro clínico para algunos autores es entre la segunda y tercera década de $v i d a^{3,4}$. Suele encontrarse afectado el sesamoideo medial o tibial, siendo escasas las descripciones que relacionan con esta patología al sesamoideo lateral o fibular y aún más infrecuentes los casos de osteonecrosis en ambos sesamoideos. Por sexos todos los autores coinciden en señalar que se da con más frecuencia en el sexo femenino ${ }^{5}$.

\section{ANATOMIA Y DESARROLLO EM- BRIOLÓGICO}

Los sesamoideos del Hallux aparecen entre la séptima y décima semana de vida intrauterina, como dos pequeñas islas de células mesenquimales dentro del tendón del flexor corto del hallux. Se produce cartílago alrededor de la doceava semana de gestación pero la osificación es tardía y no comienza hasta los 7 u 8 años de vida, pudiéndose prolongar hasta las 12 años que es cuando se completa la misma, siendo más temprana en la mujer que en el hombre ${ }^{6}$. Con frecuencia en múltiples centros de osificación, lo que puede dar sesamoideos bipartidos y tripartidos. El sesamoideo fibular o lateral rara vez es bipartido, mientras que el sesamoideo tibial o medial es bipartido en el $10 \%$ de la población, y el en $25 \%$ de éstos, es bilateral ${ }^{7}$. 
El sesamoideo fibular aparece primero en torno al día 75 seguido del sesamoideo tibial ${ }^{8}$.

El sesamoideo tibial, que suele ser más grande que el fibular se encuentra en la faceta medial de la primera cabeza metatarsal y se encuentra más impactado por la carga que el lateral, el cual se encuentra en la faceta lateral. Esta disposición anatómica le hace tener una mayor incidencia a traumatismos. Los sesamoideos del hallux son huesos constantes, pero esta descrita una serie de casos de ausencia congénita de los mismos, como una patología rara e infrecuente?

Los huesos sesamoideos, que se encuentran en la primera articulación metatarsofalángica, con sus inserciones musculares, que son el flexor corto del primer dedo, con su fascículo medial y lateral, el adductor del primer dedo, con su fascículo oblicuo y transverso, forman parte del complejo sesamoideo del hallux. La unión de los tendones de estos músculos en la inserción son un fuerte vínculo de los sesamoideos con la base de la falange proximal. Ambos sesamoideos presentan una carilla articular dorsal de cartílago hialino que articula con la cara plantar del cartílago de la cabeza del metatarsiano. Los elementos que completan el complejo sesamoideo del hallux son: el ligamento intersesamoideo, diferentes fascículos de fascia hacia ambos sesamoideos, el tendón del flexor largo del primer dedo y el ligamento intermetatarsiano ${ }^{10}$.

\section{VASCULARIZACIÓN}

El número de ramas arteriales presentes puede determinar el tiempo que tarda la consolidación de las fracturas y la incidencia de necrosis avasculares después de un traumatismo. La presencia de múltiples ramas arteriales puede proteger a un sesamoideo lesionado, mientras que la presencia de una sola rama arterial que irriga el sesamoideo dañado lo expone a riesgo de que esta pueda quedar ocluida por una fractura o por una lesión, lo que retarda la consolidación o da lugar a una pseudoarticulación.

Petterklieber y Wanivenhaus estudiaron la circulación arterial de los sesamoideos en piezas cadavéricas. En 29 disecciones estudiadas mediante arteriografía observaron tres tipos de circulación arterial. El tipo más frecuente $(52 \%)$, el tipo A, es aportada por ramas arteriales de la arteria plantar medial y del arco plantar. El tipo menos frecuente (24\%), el tipo B, se caracteriza por un patrón de riego arterial procedente primordialmente del arco plantar. Y el tipo C (24\%), es aportada por una sola rama de la arteria plantar medial. Estos autores concluyeron que el curso y la distribución del riego arterial hacia los sesamoideos podría participar en el desarrollo de una necrosis avascular tras una lesión ${ }^{11}$.

Sobel y cols., en un estudio sobre la vascularización de los sesamoideos, evaluaron el riesgo vascular de los sesamoideos medial y lateral. El mayor flujo vascular llega a la cara proximal y plantar de los sesamoideos, y un menor riesgo arterial llega al polo distal de estos. El riego vascular distal viene dado por las uniones capsulares, que aportan en la mayoría de casos, un escaso riego sanguíneo arterial. El aporte sanguíneo arterial proximal, que llega a través del flexor corto del primer dedo, irriga entre un tercio y dos tercio del sesamoideo proximal. Existen anastomosis vasculares entre el riego proximal y el proveniente de la superficie plantar que irriga el cuerpo del sesamoideo. La porción distal del sesamoideo recibe la menos cantidad de riego sanguíneo, lo que pueda dar lugar a un retraso en la consolidación o una consolidación insatisfactoria tras una lesión ${ }^{12}$.

En un estudio más reciente Bjoern y cols., en un estudio sobre la microvasculatura de los sesamoideos del hallux, determinaron que el aporte vascular principal de los sesamoideos provenía de la arteria metatarsal plantar del primer dedo y no tanto de la arteria plantar medial como decía Chamberlandy cols (1993), coincidiendo más con lo que decían Sobel y cols (1992) y Pretterklieber Wanivenhaus (1992). Además encontraron de forma frecuente una anastomosis entre la arteria plantar medial y la rama medial de la arteria metatarsal plantar del primer dedo. Y describieron la irrigación distal de los sesamoideos por parte de la arteria metatarsal plantar del primer dedo, que no había sido descrita antes en contraste a lo que decían los anteriores autores sobre la mala irrigación de la parte distal del sesamoideo. 


\section{BIOMECÁNICA}

Los sesamoideos juegan un papel importante en el funcionamiento del hallux y por lo tanto en la marcha. Están incluidos en los tendones del flexor corto del hallux y durante la fase de apoyo de la marcha juegan un papel protector de la primera metatarsofalángica y del flexor largo del hallux al absorber los impactos y dispersar las fuerzas. El complejo sesamoideo soporta normalmente en torno al $50 \%$ del peso corporal, y durante la fase de despegue puede llegar a soportar una carga de hasta $300 \%$.(13) También desempeñan una función en la fase de despegue de la marcha, aumentando su brazo de palanca, disminuyendo las fuerzas tensiles que soportan los tendones flexores y mejorando su trabajo mecánico. Al absorber la mayor parte del peso del hallux, ayudan a mejorar el impulso mecánico de la musculatura intrínseca del primer radio ${ }^{10}$.

\section{ETIOLOGÍA}

La etiología de esta patología es aún desconocida. La posición del sesamoideo tibial directamente plantar a la cabeza del primer metatarsiano somete al mismo a soportar la mayoría de las fuerza y lo predispone a sufrir patología. Por otro lado, la posición del sesamoideo lateral le permite introducirse en el espacio intermetatarsal reduciendo la carga recibida. Es por eso que algunos autores han reflejado una mayor incidencia en el sesamoideo medial ${ }^{2,13}$. Peterklieber coincidía con esto y añade que la sobrecarga repetida durante las actividades diarias podían causar un trastorno en el aporte vascular de los sesamoideos ${ }^{11}$. Kliman y cols. creían que el desarrollo de una osteocondritis es secundario a un traumatismo y posiblemente a una fractura de estrés ${ }^{14}$. Concluyendo que el proceso de reparación de la fractura de estrés podía ser la causante de la isquemia. McBryde y Anderson ${ }^{15}$ creyeron que la osteocondritis podría ser la patología inicial o bien seguida de un estrés repetido añadiendo además que cualquier fractura de estrés podía progresar hacia una osteocondritis.

Dennis y Mckinley defendían que eran más susceptibles de sufrir patología cualquier pie con una deformidad en cavo o con una plantarflexión rígida del primer metatarsiano concluyendo que era más común ver esta patología en mujeres de entre 18 y 25 años de edad y también más común en sesamoideos bipartitos ${ }^{4}$.

\section{DIAGNÓSTICO Y DIAGNÓSTICO DI- FERENCIAL}

Los pacientes con esta patología no siempre van a presentar dolor localizado en los sesamoideos, pueden referir dolor en toda la zona alrededor de la primera articulación MTF o pueden referir dolor después de un salto o golpe repentino durante la carrera. Se quejarán fundamentalmente en la fase de despegue de la marcha. Si tiene el metatarsiano en plantarflexión se puede acompañar de queratosis bajo la primera cabeza. Si el nervio digital se comprime bien por el edema, inflamación o algún fragmento del sesamoideo bipartido o fracturado puede referir síntomas neuríticos y entumecimiento $^{7}$. Otro síntoma común será dolor con la movilización de la articulación en flexión dorsal y plantar contra resistencia. Se acompañará de signos locales de inflamación, como rubor, calor, edema. McBride y Anderson sugirieron el uso de anestésico intracapsular para ayudar en el diagnóstico. Ellos creían que esto podía ayudar a diferenciar si el problema era intra o extra capsular. El bloqueo intracapsular no neutraliza todo el dolor del sesamoideo y no es fiable para hacer un diagnóstico ${ }^{15}$. Para algunos autores como Renander el diagnóstico ha de basarse en hallazgos radiográficos al considerar que apenas existen hallazgos clínicos ${ }^{2}$.

Por lo general, el diagnóstico de la osteocondritis se va a realizar mediante pruebas de imagen, principalmente la radiografía. La radiografía es la prueba complementaria de elección. La proyección axial de sesamoideos es considerada como la que mayor información aporta al permitir ver los sesamoideos aislados del resto de estructuras óseas del pie. No obstante, las proyecciones oblicua lateral, oblicua medial y lateral en descarga con dorsiflexión de los dedos también permiten visualizar los sesamoideos de forma clara ${ }^{16}$.

Las imágenes radiográficas características de esta enfermedad son, entre otras, el aspec- 
to moteado, que sugieren la presencia de áreas quísticas asociadas a zonas de esclerosis ósea; la multifragmentación del sesamoideo, la presencia de áreas con mayor densidad ósea y en los procesos muy evolucionados, imágenes compatibles con artrosis, como la deformidad de la carilla articular acompañada de disminución o pérdida del espacio articular. Sin embargo, estos signos radiológicos no se hacen evidentes hasta que el proceso patológico lleva instaurado un tiempo que oscila entre los 6 meses y los 12 meses. Durante la fase inicial no se evidencian signos de alteración sesamoidea en la valoración radiológica, pudiendo confundirse con mucha facilidad con un proceso inflamatorio de las partes blandas adyacentes a los sesamoideos (bursitis, tendinitis del FxLH) o con una metatarsalgia de tipo mecánico. En esta fase sería necesaria, la utilización de otros medios diagnósticos como RMN para realizar un correcto diagnóstico diferencial ${ }^{13}$. Las radiografías del otro pie pueden ser de ayuda para distinguir una fractura de un sesamoideo bipartito. En el sesamoideo sintomático muestra más separación de los fragmentos o unos bordes peor definidos que en el hueso del adyacente que en el lado asintomático, entonces se debe sospechar de una fractura de estrés o de un mayor trastorno de los sesamoideos bipartidos $^{17}$.

La RMN es altamente sensible a los cambios que se producen en el hueso durante la fase de isquemia ósea. Su utilidad diagnóstica en esta fase es muy similar a la que tiene la gammagrafía, aunque algunos autores señalan que la $\mathrm{RM}$ es la prueba diagnóstica más sensible a los cambios óseos iniciales ${ }^{10,13,16}$. Los hallazgos que se observan en T1 son disminución de la intensidad de la señal en el hueso secundaria a la sustitución que se produce del tejido graso y de elementos hematopoyéticos por tejido fibroso.

La gammagrafía ósea con Tc99 (Tecnecio-99) ha demostrado ser de gran utilidad en la detección de lesiones necróticas en hueso antes de su aparición radiográfica. Se observa un aumento de captación del isótopo en zonas de revascularización, con ausencia de captación en zonas de necrosis siendo la imagen característica un núcleo central frío rodeado de zona de hipercaptación. Su uso es especialmente útil cuando se pretende diferenciar las divisiones congénitas de los sesamoideos (sesamoideos bipartitos, tripartitos o multipartitos) de procesos patológicos, especialmente de fracturas de estrés y de osteonecrosis.

La tomografía axial computerizada (TAC) también permite detectar cambios en el modelo trabecular del sesamoideo durante las fases iniciales de la enfermedad, cuando aún los hallazgos radiográficos no han hecho acto de presencia. No obstante, los hallazgos descritos en las fases iniciales de la enfermedad pueden también ser observados en pacientes que han sufrido una fractura de estrés, por lo que no pueden ser considerados patognomónicos de un proceso necrótico. Así mismo, los descritos en las fases tardías de la enfermedad, guardan gran similitud con los observados en fracturas antiguas de sesamoideos, resultando muchas veces difícil determinar si la imagen del TAC se corresponde con esta patología o con una necrosis. El diagnóstico diferencial entre estas dos patologías se complica aún más si tenemos en cuenta que muchas fracturas de sesamoideos lesionan el aporte vascular al hueso y desencadenan un proceso necrótico secundario ${ }^{14}$.

En consecuencia, los resultados de las pruebas de diagnóstico por imagen siempre deberán ser interpretados a la luz de los datos aportados por la valoración clínica y por otras pruebas complementarias

Han de tenerse en cuenta muchas condiciones en el diagnóstico diferencial de la osteocondritis de sesamoideos del hallux. Estas condiciones incluyen: variaciones congénitas, hallux valgus y metatarsus primus varus, enfermedades sistémicas, infección, traumatismo previos, patología del sesamoideo interfalángico, bursitis, luxación medial del sesamoideo medial tras una cirugía de hallux valgus, condromalacia, artritis degenerativa y osteomielitis hematógena ${ }^{13}$.

\section{TRATAMIENTO}

El tratamiento inicialmente debería ser conservador. Se han descrito muchas formas de tratamiento conservador, principalmente calzado de suela gruesa con plantilla para disminuir la fuerza de carga que recae sobre el sesamoideo 
afectado. Esto se puede combinar con reposo funcional, antiinflamatorios no esteroideos y terapia física. Está descrito la aplicación de infiltraciones con corticoides, pero en ocasiones nos pueden dar el efecto contrario porque al paciente no le duele y siguen cargando la zona. Los soportes deberán usarse por lo menos 6 meses, aunque se resuelva rápido, y se deberán volver a usar si los síntomas recurren después de los 6 meses. Axe y Ray ${ }^{18}$ exponen que el tratamiento ortopédico es efectivo en el atleta con dolor en los sesamoideos sin importar la etiología. Axe y Ray ${ }^{18}$ proponen el uso de una ortesis funcional que controle el movimiento de la articulación subastragalina y disminuyan la presión sobre los sesamoideos mediante un "cut out". En su estudio ocho de cada diez atletas tratados con ortesis a medida sintieron alivio del dolor sesamoideo. La ventaja del tratamiento ortopédico es que permite que el atleta continúe su participación sin pérdida de práctica o tiempo de competición y retrasa la necesidad de intervención quirúrgica. Si nos encontramos ante una fractura de estrés ya sea de sesamoideos bipartidos o no, se puede intentar tratamiento conservador, pero, según Richardson ${ }^{16}$, no siempre es efectivo. La fractura de estrés puede ser difícil de diagnosticar en ausencia de una amplia separación entre fragmentos y formación de callo óseo, pero se debe de sospechar en el atleta si continúa con síntomas a pesar de un largo descanso y un manejo conservador. Puesto que la presencia de aspecto aplanado, moteado, fragmentado, en el sesamoideo sintomático del atleta indica probablemente una oscteocondritis, Richardson cree que periodos muy largos de tratamiento conservador no están justificados y no son efectivos. Si los síntomas de fracturas desplazadas o no desplazadas, sesamoiditis, osteocondrosis o osteocondritis no se resuelven de 3 a 6 meses con tratamiento conservador, él recomienda escisión quirúrgica del sesamoideo.

La mayoría de autores coinciden en que el tratamiento quirúrgico no se debe considerar, hasta que se haya agotado todas las posibilidades de tratamiento conservador ${ }^{7,15,17-19}$, incluyendo plantilla, modificaciones de calzado, diminución de la carga o ausencia de la misma e inmovilización mediante bota o yeso.

El tratamiento quirúrgico de la patología dolorosa de los sesamoideos del hallux incluye la resección parcial o completa de uno o de ambos sesamoideos. Se debe evitar la escisión de ambos sesamoideos debido a la gran incidencia post quirúrgica de hallux valgus y dedo en garra. Además, va a producir un defecto mecánico en el flexor corto del hallux, reduciendo el momento de flexión plantar de la articulación metatarsofalángica ${ }^{7}$.

El abordaje quirúrgico dependerá de que sesamoideo se quiera resecar.

\section{SESAMOIDECTOMÍA TIBIAL}

Cuando esté indicada la sesamoidectomía tibial de forma aislada, se recomienda una incisión longitudinal en la cara medial, entre las ramas digitales plantar y dorsal. Las ventajas de este abordaje son que se evita la incisión plantar, buena exposición, menos riesgo de contractura de la articulación metatarsofalángica y el evitar los nervios digitales plantares. Las desventajas de esta incisión son su proximidad al paquete neurovascular plantar-medial y la posibilidad de una cicatriz dolorosa que puede irritarse con el roce del zapato ${ }^{20}$.

Técnica: Se realiza incisión medial de $3 \mathrm{~cm}$. Se identifica la rama plantar digital y se retrae para evitar dañarla. Se localiza el sesamoideo mediante la palpación diferenciándolo de la cabeza metarsal. Con el hallux flexionado a 20-30 grados y el flexor largo retraído, se incide el ligamento intersesamoideo y se tira del sesamoideo tibial hacia medial. Se reseca con disección cortante separándolo de la cápsula y placa plantar mediante un bisturí pequeño. La escisión se lleva a cabo mediante una liberación proximal del fascículo medial del flexor corto del hallux y su continuación distalmente a la base de la falange proximal. Se sutura la cápsula medialmente con sutura absorbible y la piel se sutura no absorbible ${ }^{7}$.

\section{SESAMOIDECTOMIA FIBULAR}

Para la escisión del sesamoideo lateral se puede hacer un abordaje dorsal o plantar. El abordaje dorsal requiere mucha habilidad debido a la profundidad del sesamoideo, pero se previene la aparición de posibles ciatrices dolorosas en la planta, sin embargo, con el abordaje plantar la presencia del paquete neurovascular del primer espacio dificulta la escisión. Existe cierta polémica con las incisiones plantares debido a 
que ocupan una superficie de carga. Las desventajas incluyen la posibilidad de cicatriz hipertrófica, la necesidad de no apoyar el pie durante más de 4 semanas y la interrupción de la grasa plantar. Las ventajas de este abordaje son que permite un acceso más fácil, visualización directa del tendón del flexor largo del hallux ${ }^{20}$.

Técnica dorsal: Se realiza incisión dorsal en el primer espacio intermetatarsal. Las ramas del nervio peroneo profundo se identifican y se protege. Se desprende el tendón del adductor de la cápsula articular y se expone el sesamoideo peroneo. A continuación se, se diseca el tendón del adductor y se separa de la cara lateral del sesamoideo, y después se secciona el ligamento intersesamoideo. Se desplaza el sesamoideo más hacia lateral y se libera en su parte distal y proximal y después se escinde. Se debe inspeccionar la profundidad de la herida para asegurar que el tendón flexor largo del dedo gordo no se ha seccionado y que el paquete neurovascular está conservado. Además hay que comprobar si se ha desprendido el tendón del adductor y en ese caso reinsertarlo. Se sutura la piel.

Técnica plantar. Se realiza incisión longitudinal, comenzando de 1 a $1,5 \mathrm{~cm}$ distal a la articulación metatarsofalángica, extendiéndola proximalmente de 3.5 a $4 \mathrm{~cm}$ entre el primer y segundo metatarsiano ${ }^{7}$. Richard Bouche y cols. Basándose el estudio de Pretterkleiber y Wanivenhaus de la anatomía vascular de los sesamoideos previamente citado, recomiendan una incisión longitudinal entre los sesamoideos para sesamoidectomías fibulares aisladas o combinadas de tibial y fibular, añadiendo que las otros abordajes pueden interrumpir el aporte vascular de los sesamoideos ${ }^{20}$. Una vez separados piel, fascia y tejido graso, se introduce un retractor. Con la ayuda de unas tijeras de disección pequeñas de punta roma, se retrae el paquete neurovascular hacia un lado o el otro dependiendo de la posición del sesamoideo. La polea encima del flexor largo del hallux se abre, y se retrae el tendón medialmente. En este punto, se podrá observa el ligamento intersesamoideo, el cual hay que seccionarlo completamente. Se sujeta el sesamoideo fibular con un mosquito pinza y se libera de la inserción lateral del flexor cor- to del hallux en su parte proximal en visión directa. Una vez liberado en su parte medial y proximal, se desinserta el adductor de su parte lateral y distal. La última fijación del hueso sesamoideo se secciona distalmente donde la placa plantar continúa su inserción distal en la falange proximal. Una vez retirado el sesamoideo, se inspecciona la herida ${ }^{7}$.

\section{CONCLUSIÓN}

A pesar de que la osteocondritis de los sesamoideos es una patología infrecuente, puede presentarse en una consulta de podología por lo que debe ser incluida en el diagnóstico diferencial de metatarsalgias y dolor en antepié. La baja incidencia de la misma nos puede llevar a un diagnóstico erróneo y como consecuencia a un retraso en el tratamiento de algunos pacientes. Su diagnóstico requiere un alto nivel de destreza y un examen físico cuidadoso dirigido a la ubicación específica del dolor.

Sospecharemos de una osteocondritis sobretodo en jóvenes atletas, deportistas, bailarines de ballet de entre 20 y 30 años. Si la clínica nos hace pensar que se trata de una osteocondritis, nos ayudaremos de pruebas de imagen para confirmar el diagnóstico como radiografía simple, resonancia, gammagrafía o TAC.

El tratamiento no varía significativamente con respecto al empleado en otras patologías de sesamoideos del hallux. La mayoría de autores defienden que la osteocondritis debe tratarse de un modo conservador, para evitar así una intervención quirúrgica. Se necesitan más estudios a largo plazo para determinar la tasa de incidencia y recurrencia de esta patología.

$\mathrm{Si}$ el tratamiento conservador no es efectivo está indicado la escisión del sesamoideo necrótico. El tratamiento quirúrgico puede resultar beneficioso, pero siempre hay que avisar al paciente de la posibilidad de nuevas deformidades como hallux varus y garra.

En cuanto al abordaje quirúrgico, en el sesamoideo medial los autores coinciden en el abordaje medial, pero en el sesamoideo lateral, parece no haber consenso entre el abordaje dorsal o plantar. 


\section{BIBLIOGRAFÍA}

1. Atanda A, Shah SA, O'Brien K. Osteochondrosis: common causes of pain in growing bones. Am Fam Physician . $2011 ; 83(3): 285-91$

2. Renander A. Two Cases of Typical Osteochondropathy of the Medial Sesamoid Bone of the First Metatarsal. Acta Radiol. 1924;3(6):521-7.

3. Wuelker N, Wirth CJ. The great toe sesamoids. Foot Ankle Surg. 1996;2(3):167-74.

4. Dennis KJ, McKinney S. Sesamoids and accessory bones of the foot. Clin Podiatr Med Surg. 1990;7(4):717-23.

5. Julsrud ME. Osteonecrosis of the tibial and fibular sesamoids in an aerobics instructor. J Foot Ankle Surg. 1997 ;36(1):31-5.

6. Karadaglis D, Grace D. Morphology of the hallux sesamoids. Foot Ankle Surg. 2003;9(3):165-7.

7. Richardson EG. Hallucal sesamoid pain: causes and surgical treatment. J Am Acad Orthop Surg.;7(4):270-8.

8. Brenner E, Gruber H, Fritsch H. Fetal development of the first metatarsophalangeal joint complex with special reference to the intersesamoidal ridge. Ann Anat. 2002;184(5):481-7.

9. Day F, Jones PC, Gilbert CL. Congenital absence of the tibial sesamoid. J Am Podiatr Med Assoc. 2002;92(3):153-4.

10. Garrido IM, Bosch MN, González MS, Carsí VV. Osteochondritis of the hallux sesamoid bones. Foot Ankle Surg. 2008 Jan;14(4):175-9.

11. Pretterklieber ML, Wanivenhaus A. The arterial supply of the sesamoid bones of the hallux: the course and source of the nutrient arteries as an anatomical basis for surgical approaches to the great toe. Foot Ankle. 1992 Jan;13(1):27-31.

12. Sobel M, Hashimoto J, Arnoczky SP, Bohne WH. The microvasculature of the sesamoid complex: its clinical significance. Foot Ankle.;13(6):359-63.

13. Fleischli J, Cheleuitte E. Avascular necrosis of the hallucial sesamoids. J Foot Ankle Surg. 1995;34(4):358-65.

14. Kliman ME, Gross AE, Pritzker KP, Greyson ND. Osteochondritis of the hallux sesamoid bones. Foot Ankle.;3(4):220-3.

15. McBryde AM, Anderson RB. Sesamoid foot problems in the athlete. Clin Sports Med. 1988;7(1):51-60.

16. Richardson EG. Injuries to the hallucal sesamoids in the athlete. Foot Ankle. 1987;7(4):229-44.

17. Ilfeld FW, Rosen V. Osteochondritis of the first metatarsal sesamoid: report of three cases. Clin Orthop Relat Res. 1972;85:38-41.

18. Axe MJ, Ray RL. Orthotic treatment of sesamoid pain. Am J Sports Med.;16(4):411-6.

19. Jahss MH. The sesamoids of the hallux. Clin Orthop Relat Res. 1981 ;(157):88-97.

20. Bouché R, Heit E. Surgical approaches for hallucal sesamoid excision. J Foot Ankle Surg. 2002;41(3):192-6. 